\title{
Rapid Determination of Chiral Amino Acids by LC-Q-TOF Mass Spectrometry
}

\author{
Sooyeul Cho, " Insun Lee, Jinho Kim, In Sun Hwang, Sungil Kim, Kyoung Moon Han, \\ Ji Won Kim, Ji Hyun Lee, Yong Keun Park, ${ }^{\dagger}$ Soon Young Han, and Kab Ryong Chae \\ Toxicological Evaluation and Research Department, National Institute of Food and Drug Safety Evaluation, \\ Korea Food \& Drug Administration, Osong Health Technology Administration Complex, \\ Chungcheongbuk-do 363-951, Korea. "E-mail: chos219@korea.kr \\ 'Department of Life Science \& Biotechnology, Korea University, Korea \\ Received October 9, 2011, Accepted October 21, 2011
}

Key Words : Isomer, Amino acid, HPLC, LC/TOFMS

The discovery of molecular asymmetry by Pasteur in 1848 established the foundation for our understanding of molecular chirality. Recently, the interest in safe food and pharmaceuticals has been increasing owing to increase of elderly population with longer average life expectancy according to improvements in income levels and development of medical technology. Isomers are compounds with the same molecular formula but different dimensional structures and dissimilar properties. ${ }^{1-3}$ While as a isomer in racemate consisted of two isomers in pharmaceuticals is predicted of biological activities, the other does not show the biological activity. ${ }^{4}$ The separation of the cis- or trans- fatty acids in oil and the separation of the L- or D-amino acids in protein is being watched with interesting food industry. ${ }^{5}$

The case of the racemic drug thalidomide is worthy of comment. It was already reported that $R$-isomer of thalidomide was activated for a sleeping pill, whereas $S$-isomer resulted in the birth of deformed babies. This negative factor of racemic drug has been led to an increasing preference for single enantiomers in both industry and regulatory authorities. Regulatory control of chiral drugs began in the US with the publication in 1992 of formal guidelines on the development of chiral drugs in a document entitled Policy statement for the Development of New stereoisomeric Drugs. ${ }^{6,7}$

Essential amino acids are classified by L- and D-amino acid. L-amino acids are generally used in feed additives, food and pharmaceutical ingredients, while D-amino acids are applicable for therapeutic raw materials. Essential amino acids enantiomers show different bioactivity according to amino acid ${ }^{8-12}$ like an increase of serotonine by L-tryptophan, part precursor of taurine and cystein and so on. L-amino acid can be consumed through food but D-amino acid is different pathway of produce and feed (e.g D-amino acids are produced by artificial synthesis)

A number of approaches have been used for studying the chiral recognition of organic compounds in the solution phase, which include circular dichroism, capillary electrophoresis, nuclear magnetic resonance, use of chiral reagents in chromatography, etc.. ${ }^{13}$ However, it is difficult and complex for such approaches to be applied to products available in the market. This study was conducted to detect products which contain side-effect causing isomer by presenting a method to identify enantiomers of amino acid present in food and drug products.

This research was to study a method to separate single enantiomer from racemate using HPLC and LC-Q-TOF mass spectrometry and we developed and validated the separation analytical method of isomers in amino acid (tryptophan, methionine, phenylalanine, histidine).

To establish method is the best condition to separate of L-, D-phenylalanine, tryptophan, methionine and histidine on simultaneous analytical chromatogram by using HPLC and LC-Q-TOF mass spectrometry (Fig. 1). The retention time of D-isomer is longer than their corresponding L-isomer which is similar to results obtain by G. Wu, M. Furlanut. ${ }^{18}$ The resolution (Rs) of the best separation condition was established L- and D-phenylalanine: 1.898, L- and Dtryptophan: 1.797 , L- and D-methionine: 2.170 , L- and Dhistidine: 1.112. Parents molecular ion were confirmed in each of MS spectrum. The resolution of peak in LC-Q-TOF mass spectrometry was L- and D-phenylalanine: 1.561 , Land D-tryptophan: 1.644, L- and D-methionine: 1.217, L- and D-histidine: 1.301.

We worked to validate the effectiveness of this analytical method by checking linearity, precision, accuracy, limit of detection, limit of quantification and stability. The correlation coefficient $\left(\mathrm{R}^{2}\right)$ was up to 0.999 at 5 point concentration (Table 1). As shown in Table 2, LOD was 0.30-1.50 $\mathrm{mg} / \mathrm{L}$ and LOQ was $0.80-2.00 \mathrm{mg} / \mathrm{L}$ with HPLC, and LOD was $16.5-250.0 \mu \mathrm{g} / \mathrm{L}$ and LOQ was $25.0-500.0 \mu \mathrm{g} / \mathrm{L}$ with LC-Q-TOF mass spectrometry, respectively. It is difference LOD and LOQ as component in same condition according to HPLC and LC-TOF mass spectrometry.

In order to check precision and accuracy of analytical substances, we conducted repeated tests of 5 different concentrations three times a day according to validation of guidelines. In addition, we performed repeated tests of each concentration three times over the course of three days. Precision was calculated as RSD \% by dividing standard deviation of peak concentration by peak concentration mean value while accuracy calculated as RSD \% through dividing mean value estimated based on calibration curve by substances concentration. 
(a)
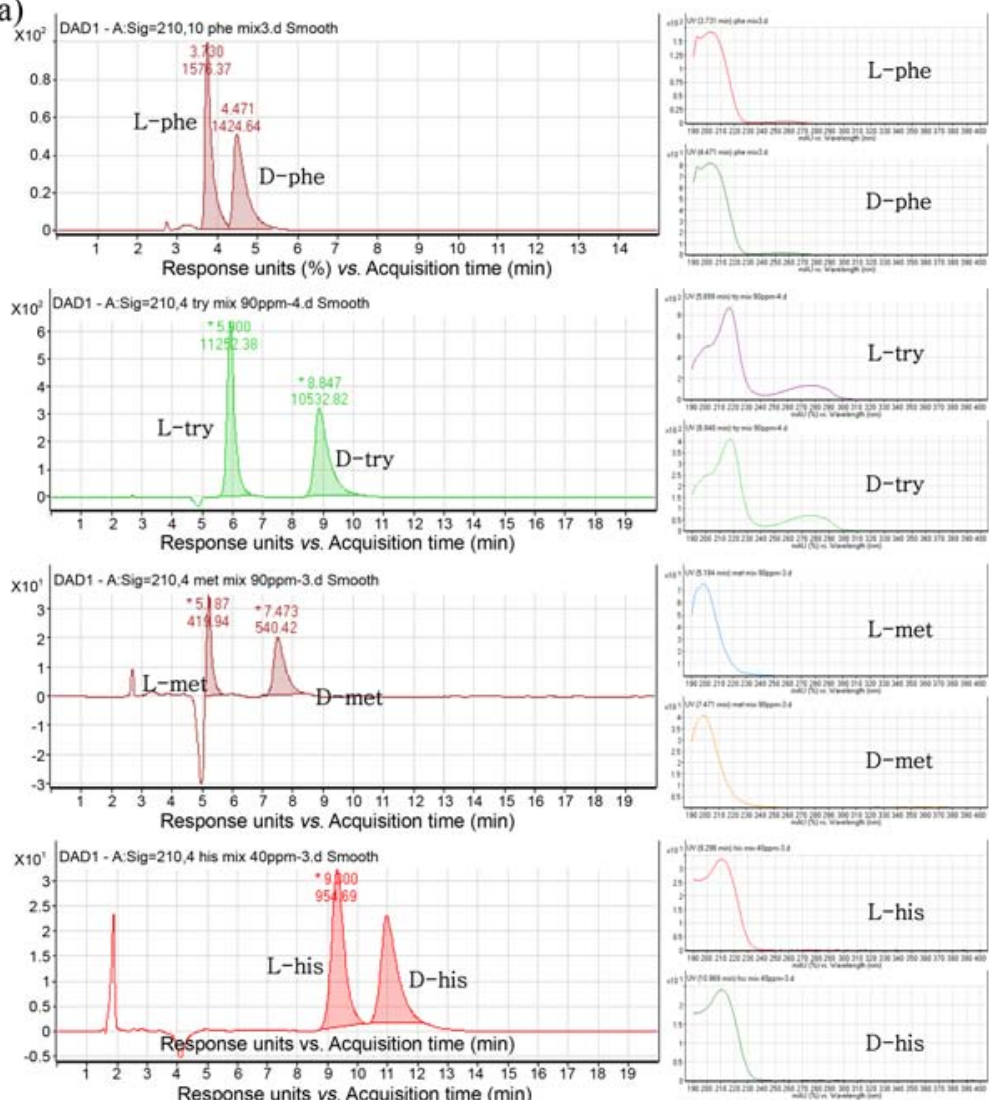

(b)
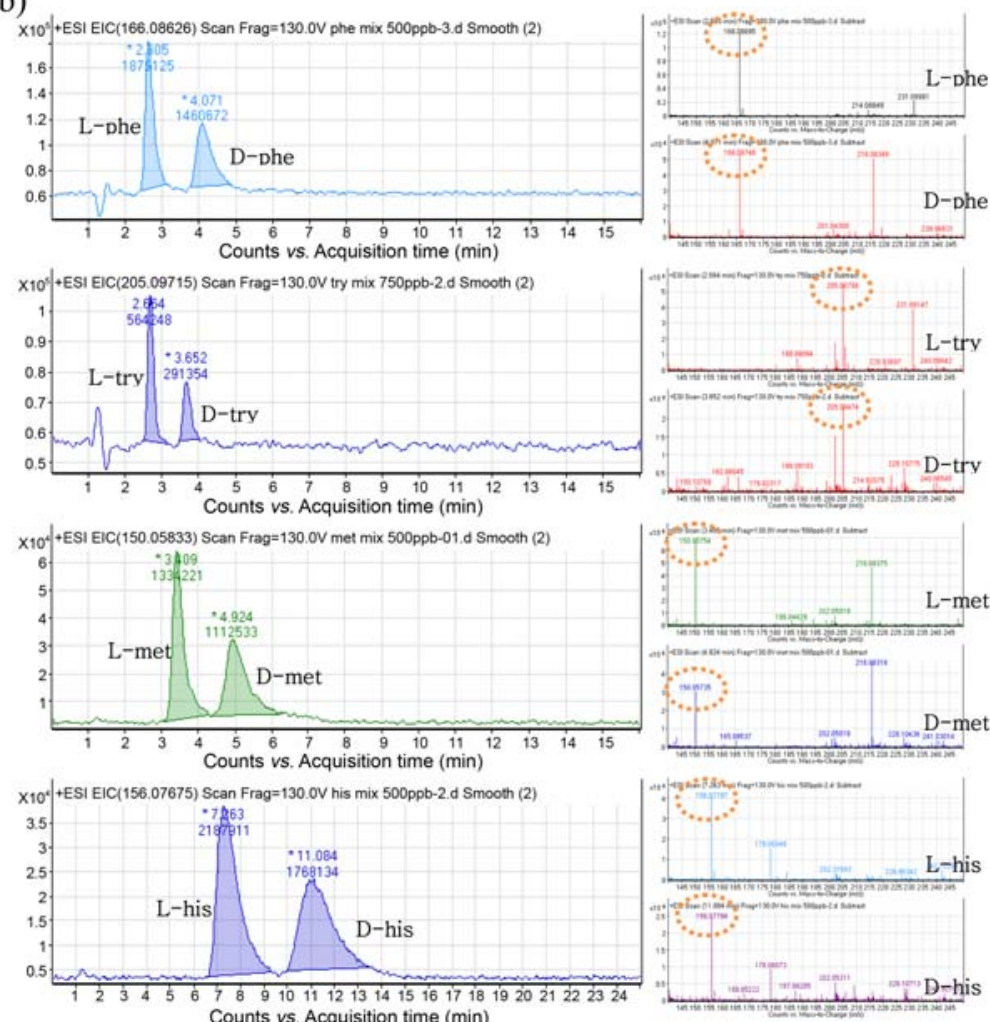

Figure 1. The simultaneous analytical chromatogram of 8 amino acids by analyzing HPLC and LC/TOFMS. To establish method is the best condition to separate of chiral amino acids. Molecular ion of each amino acids is phenylalanine 166.08626, tryptophan 205.09715, methionine 150.05883 and histidine 156.07675. (a) The simultaneous analytical chromatogram and PDA spectrum of L- and D- amino acids analyzing by HPLC (b) The simultaneous analytical EIC chromatogram and MS spectrum of L- and D-amino acids analyzing by LC/TOF MS. 
Table 1. Linear regression data, LOD and LOQ of investigated compounds by analyzing HPLC and LC/TOF MS

\begin{tabular}{|c|c|c|c|c|c|c|c|c|}
\hline & \multicolumn{4}{|c|}{ HPLC (mg/L) } & \multicolumn{4}{|c|}{ LC/TOF MS (ug/L) } \\
\hline & $\mathrm{R}^{2}$ & Linear range & LOD & LOQ & $\mathrm{R}^{2}$ & Linear range & LOD & LOQ \\
\hline L-phenylalanine & 0.9998 & $2.5 \sim 100$ & 1.50 & 2.00 & 0.9994 & $30 \sim 500$ & 20.0 & 30.0 \\
\hline D-phenylalanine & 0.9999 & $2.5 \sim 100$ & 0.75 & 1.50 & 0.9995 & $100 \sim 500$ & 66.5 & 75.0 \\
\hline L-tryptophan & 0.9998 & $2.5 \sim 100$ & 0.30 & 0.80 & 0.9983 & $500 \sim 2000$ & 250.0 & 500.0 \\
\hline D-tryptophan & 0.9998 & $2.5 \sim 100$ & 0.30 & 0.80 & 0.9977 & $500 \sim 2000$ & 200.0 & 500.0 \\
\hline L-methionine & 0.9996 & $2.5 \sim 100$ & 1.50 & 2.00 & 0.9995 & $25 \sim 500$ & 16.5 & 25.0 \\
\hline D-methionine & 0.9999 & $2.5 \sim 100$ & 0.50 & 1.00 & 0.9994 & $75 \sim 500$ & 50.0 & 75.0 \\
\hline L-histidine & 0.9993 & $2.5 \sim 100$ & 0.30 & 0.80 & 0.9993 & $75 \sim 500$ & 50.0 & 75.0 \\
\hline D-histidine & 0.9991 & $2.5 \sim 100$ & 0.50 & 0.80 & 0.9963 & $100 \sim 1000$ & 75.0 & 100.0 \\
\hline
\end{tabular}

Table 2. The amino acid results of precision and accuracy by analyzing HPLC and LC/TOF MS

\begin{tabular}{|c|c|c|c|c|c|}
\hline & & \multicolumn{2}{|c|}{ HPLC (\%) } & \multicolumn{2}{|c|}{ LC/TOF MS (\%) } \\
\hline & & Precision & Accuracy & Precision & Accuracy \\
\hline \multirow[t]{2}{*}{ L-phenylalanine } & intra-day & $0.04 \sim 0.98$ & $98.2 \sim 109.5$ & $0.39 \sim 2.20$ & $90.8 \sim 108.5$ \\
\hline & inter-day & $0.04 \sim 1.41$ & $98.2 \sim 110.9$ & $0.17 \sim 1.61$ & $94.9 \sim 110.7$ \\
\hline \multirow[t]{2}{*}{ D-phenylalanine } & intra-day & $0.02 \sim 0.64$ & $97.5 \sim 106.0$ & $0.27 \sim 1.78$ & $96.3 \sim 104.0$ \\
\hline & inter-day & $0.12 \sim 0.64$ & $97.5 \sim 108.0$ & $0.22 \sim 1.90$ & $94.2 \sim 103.1$ \\
\hline \multirow[t]{2}{*}{ L-tryptophan } & intra-day & $0.05 \sim 1.04$ & $96.7 \sim 115.6$ & $1.05 \sim 1.72$ & $94.8 \sim 106.7$ \\
\hline & inter-day & $0.01 \sim 0.71$ & $97.1 \sim 109.2$ & $0.19 \sim 2.05$ & $96.2 \sim 107.9$ \\
\hline \multirow[t]{2}{*}{ D-tryptophan } & intra-day & $0.01 \sim 0.42$ & $97.2 \sim 118.1$ & $0.42 \sim 3.20$ & $94.1 \sim 105.1$ \\
\hline & inter-day & $0.03 \sim 1.30$ & $97.1 \sim 119.6$ & $0.62 \sim 2.21$ & $95.7 \sim 106.5$ \\
\hline \multirow[t]{2}{*}{ L-methionine } & intra-day & $0.23 \sim 0.82$ & $97.2 \sim 113.6$ & $0.66 \sim 1.73$ & $94.7 \sim 106.1$ \\
\hline & inter-day & $0.07 \sim 1.49$ & $96.5 \sim 110.8$ & $0.23 \sim 1.91$ & $92.0 \sim 104.6$ \\
\hline \multirow[t]{2}{*}{ D-methionine } & intra-day & $0.46 \sim 1.57$ & $94.5 \sim 108.0$ & $0.63 \sim 2.03$ & $97.4 \sim 102.6$ \\
\hline & inter-day & $0.63 \sim 1.17$ & $96.7 \sim 111.2$ & $0.16 \sim 1.37$ & $96.7 \sim 106.9$ \\
\hline \multirow[t]{2}{*}{ L-histidine } & intra-day & $0.21 \sim 1.57$ & $95.6 \sim 119.2$ & $1.08 \sim 1.66$ & $96.9 \sim 105.5$ \\
\hline & inter-day & $0.03 \sim 2.04$ & $95.6 \sim 119.7$ & $1.01 \sim 3.84$ & $97.0 \sim 106.5$ \\
\hline \multirow[t]{2}{*}{ D-histidine } & intra-day & $0.06 \sim 1.66$ & $94.9 \sim 119.8$ & $1.85 \sim 5.48$ & $96.2 \sim 111.6$ \\
\hline & inter-day & $0.05 \sim 2.05$ & $94.9 \sim 119.9$ & $0.83 \sim 3.08$ & $90.6 \sim 109.8$ \\
\hline
\end{tabular}

Precision and accuracy were $0.01-2.05 \%$ and $94.5-119.9 \%$ with HPLC, $0.17-5.48 \%$ and 90.6-111.6\% with LC-Q-TOF mass spectrometry according to 8 amino acids, respectively (Table 2).

The relative standard deviations for stability were less than $2 \%$ as $0.28-1.92$ and $7 \%$ as $0.62-6.98$ by analyzing HPLC and LC-Q-TOF mass spectrometry, respectively (Table 3). It was to be stable for at least 24 hours.

Table 3. The relative standard deviations for stability of amino acids by analyzing HPLC and LC/TOF MS

\begin{tabular}{lcc}
\hline & HPLC (\%) & LC/TOF MS (\%) \\
\hline L-phenylalanine & 1.44 & 0.70 \\
D-phenylalanine & 1.39 & 0.62 \\
L-tryptophan & 0.99 & 2.49 \\
D-tryptophan & 1.31 & 6.98 \\
L-methionine & 1.92 & 0.89 \\
D-methionine & 1.20 & 1.58 \\
L-histidine & 0.28 & 1.46 \\
D-histidine & 1.50 & 1.27 \\
\hline
\end{tabular}

Table 4 shows the level of suitability of L-phenylalanine, -tryptophan, -methionine, -histidine in products retailed in market by analyzing HPLC and LC-Q-TOF mass spectrometry. The results were derived in the range of 90.1 to $118.5 \%$ to 6 commercial samples containing $\mathrm{L}$-form.

The method is capable of separating enantiomers faster in a precise manner compared to other methods, so for that reason, we think that this method will be useful in deciding whether racemate is contained or when developing new drugs.

The results of this study are expected to be useful in confirming safety and effectiveness of food and drug components. It will be continuously necessary to perform the development separation methods of isomers for the advancement of food and drug industry.

\section{Experimental Section}

Materials. L-, D-phenylalanine, tryptophan, methionine, and histidine were obtained and purchased from Sigma (UK). All chemicals are of analytical grade were purchased from Sigma-Aldrich (St. Louis, MO, USA) and Merk. 
Table 4. L-phenylalanine, -tryptophan, -methionine and -histidine levels in products retailed in market by analyzing HPLC and LC/TOFMS

\begin{tabular}{|c|c|c|c|c|c|c|c|c|}
\hline \multirow{2}{*}{ No } & \multicolumn{2}{|c|}{ L-phenylalanine $($ Mean \pm SD) } & \multicolumn{2}{|c|}{ L-tryptophan (Mean \pm SD) } & \multicolumn{2}{|c|}{ L-methionine $($ Mean $\pm \mathrm{SD})$} & \multicolumn{2}{|c|}{ L-histidine (Mean $\pm \mathrm{SD}^{a}$ ) } \\
\hline & HPLC & LC/TOFMS & HPLC & LC/TOFMS & HPLC & LC/TOFMS & HPLC & LC/TOFMS \\
\hline 1 & $92.7 \pm 0.05$ & $116.8 \pm 4.19$ & $101.8 \pm 0.09$ & $101.1 \pm 3.98$ & $93.9 \pm 0.45$ & $98.2 \pm 3.92$ & $93.9 \pm 0.33$ & $93.6 \pm 0.89$ \\
\hline 2 & $90.2 \pm 0.29$ & $117.4 \pm 5.59$ & $96.2 \pm 0.05$ & $102.2 \pm 5.83$ & $91.7 \pm 0.23$ & $97.3 \pm 5.79$ & $98.5 \pm 0.25$ & $92.7 \pm 1.81$ \\
\hline 3 & $92.0 \pm 0.04$ & $116.4 \pm 6.00$ & $99.5 \pm 0.07$ & $100.5 \pm 2.77$ & $93.6 \pm 0.41$ & $90.9 \pm 4.08$ & $99.4 \pm 0.30$ & $95.8 \pm 2.95$ \\
\hline 4 & $92.6 \pm 1.95$ & $118.5 \pm 4.51$ & $99.5 \pm 0.13$ & $100.5 \pm 2.07$ & $100.0 \pm 0.12$ & $92.4 \pm 3.87$ & $100.3 \pm 0.26$ & $99.1 \pm 1.62$ \\
\hline 5 & $92.2 \pm 0.04$ & $114.8 \pm 6.00$ & $98.0 \pm 0.01$ & $91.0 \pm 2.04$ & $102.2 \pm 0.24$ & $96.0 \pm 3.59$ & $99.0 \pm 0.08$ & $92.5 \pm 2.35$ \\
\hline 6 & $92.9 \pm 0.05$ & $108.8 \pm 4.93$ & $96.6 \pm 0.10$ & $90.1 \pm 0.32$ & $94.4 \pm 0.46$ & $97.4 \pm 2.61$ & $99.6 \pm 0.36$ & $93.2 \pm 3.04$ \\
\hline
\end{tabular}

${ }^{a}$ Mean $\pm \mathrm{SD}$ : Average Amount $\pm \mathrm{SD}(\mathrm{n}=6)$

HPLC and Accurate-Mass Quadrupole Time-of-Flight (Q-TOF) LC/MS. The high-performance liquid chromatography (HPLC) system consisted of a solvent delivery system (Agilent 1200, CA, USA), an injection valve with a $10 \mu \mathrm{L}$ autosampler, a chirosil $\mathrm{RCA}(+)(4.6 \times 150,5 \mathrm{um}$, RS tech corp.) was derived from (+)-(18-crown-6)-2,3,11,12tetracarboxylic acid. The mobile phase of the chromatography was prepared by mixing methanol-water $(70: 30, \mathrm{v} / \mathrm{v})$, and phosphoric acid to reach a final concentration of 0.005 $\mathrm{g} / 100 \mathrm{~mL}$ for separating L-, D-tryptophan, phenylalanine, methionine and histidine. The mobile phase flow was $0.8 \mathrm{~mL} / \mathrm{min}$, UV detection length was $210 \mathrm{~nm}$, PDA detection range was $190-400 \mathrm{~nm}$ and the column oven was at 35 ${ }^{\circ} \mathrm{C}$.

The Accurate-Mass Quadrupole Time-of Flight (Q-TOF) LC/MS (Agilent Technologies 6530) composed of a electrospray ionization (ESI), 350 gas temperature, $65 \mathrm{~V}$ skimmer voltage and $130 \mathrm{~V}$ fragmentor voltage were controlled for MS. It was used for HPLC that column used Chirosil$\mathrm{RCA}(+)(2.1 \times 150 \mathrm{~mm}, 5 \mathrm{um}, \mathrm{RS}$ tech corp. $)$ from RS Tech. (Korea), the mobile phase of the chromatography was prepared by mixing $0.1 \%$ formic acid in distilled water and $0.1 \%$ formic acid acetonitril $(40: 60, \mathrm{v} / \mathrm{v})$, the mobile phase flow was set at $0.8 \mathrm{~mL} / \mathrm{min}$ and temperature of column was set at $20{ }^{\circ} \mathrm{C}$.

Method Validation. The methods were examined by items of specificity, linearity, precision, accuracy, limit of detection, limit of quantification and stability using FDA, ICH and USP guidelines. ${ }^{14-17}$

Acknowledgments. This work was supported by the Korea Food and Drug Administration Fund.

\section{References}

1. Fessenden, R. J., Fessenden, J. S. Fundamentals of Organic Chemistry 1998, 203-236.

2. McMurry, J. Organic. Chemistry 1996, 78-93

3. Cancer, H.; Groner, E.; Levy, L.; Agranat, I. Drug Discovery Today 2004, 9, 105.

4. Fritsche, J.; Rickert, R.; Steinhart, H.; Yurawecz, M. P.; Mossoba, M. M.; Sehat, N.; Roach, J. A. G.; Kramer, J. K. G.; Ku, Y. Fett/ Lipid. 1999, 8, 272-276.

5. Lee, S. H.; Chang, Y. H.; Lee, K. P. Analytical Science \& Technology 1996, 9, 52-61.

6. Guidance for Industry: FDA's Policy Statement for the Development of New Stereoisomeric Drugs; CDER, US FDA, 1992.

7. Subramanian, G. A Practical Approach to Chiral Separations by Liquid Chromatography 1994; p 62.

8. Rekoslavskaya, N. I. Journal of Plant Physiol. 1999, 25, 39-49

9. Obeid, O. A.; Johnston, K.; Emery, P. W. European Journal of Clinical Nutrition 2004, 58, 105-109.

10. Stryer, L. Biochemistry 4th ed, 2000; p 201-202.

11. Park, H.; Kim, K. M.; Lee, A.; Ham, S.; Nam, W.; Chin, J. J. Am. Chem. Soc. 2007, 129, 1518-1519.

12. Sabelli, H. C.; Javaid, J. I. Phenylalanine Neuropsychiatry Clin Neurosci. 1995, 7(1), 6-14.

13. Snggeeta Kumari et al., American Society for Mass Spectrometry 2007, 18, 1516-1524.

14. FDA Guidance for Industry. Analytical Procedures and Methods Validation.

15. ICH Q2A Guideline. Validation of Analytical Methods Definitions and Terminology.

16. USP29. Validation of Compendial Methods

17. Q6A International conference on Harmonization; Guidance on Q6A Specification: Test Procedures and Acceptance Criteria for New Drug Substances and New Drug Products: Chemical Substances, 2000.

18. Wu, G.; Furlanut, M. Il Farmaco 1999, 54, 188-190.

19. Lee, S. H.; Chang, Y. H.; Lee, K. P. Analytical Science \& Technology 1996, 9, 52-61.

20. Aldegunde, M.; Soengas, J. L.; Rozas, G. Journal of Experimental Zoology 2000, 286, 131-135. 